\title{
Ärztegesundheit: Eine zentrale Aufgabe der Deutschen Suchtstiftung Matthias Gottschaldt
}

Die Deutsche Suchtstiftung Matthias Gottschaldt ist benannt nach dem Mann, der als Arzt selbst an einer Alkoholabhängigkeit erkrankte und durch seinen offensiven und ehrlichen Umgang mit seiner eigenen Erkrankung für viele ein positives Leitbild war. Durch seine Überzeugungskraft als Betroffener und als Arzt gelang es ihm, das Thema Ärztegesundheit in die Gesellschaft zu tragen und die Akzeptanz von Suchterkrankungen bei Ärzten in der Gesellschaft zu erhöhen. Sucht war für ihn keine stigmatisierende Erkrankung, sondern eine Chance zur konstruktiven persönlichen Weiterentwicklung.

Ein zentrales Anliegen der Stiftung ist es daher, an einer Abhängigkeit erkrankte Ärzte zu unterstützen und deren Behandlungschancen durch adäquate Behandlungsangebote zu verbessern. Obwohl Ärzte ein erhöhtes Risiko für die Entwicklung von psychischen Störungen wie Burn-Out, Depressionen oder Suchterkrankungen haben, gibt es bis heute in Deutschland nur in einigen Bundesländern gut strukturierte Behandlungskonzepte.

Zur Verbesserung dieser Situation wurde von der Deutschen Suchtstiftung Matthias Gottschaldt im Jahr 2005 der „Expertenrat suchtgefährdete Ärztinnen und Ärzte“ gegründet. Ziel dieses Expertenrates ist es, ein Forum für alle Fragen der Prävention und Behandlung suchterkrankter Ärzte zu sein.

Ein Beispiel für ein bestehendes und erfolgreiches Behandlungsangebot für betroffene Ärzte ist das von Prof. Gottschaldt entwickelte „Curriculum für abhängige Ärzte“. Erste Ergebnisse zeigen Erfolgsraten von über $80 \%$ (siehe Artikel von Mundle „Hilfsangebote für suchtkranke Ärzte“).

Als nächsten Schritt möchte die Deutsche Suchtstiftung Matthias Gottschaldt eine entsprechende Plattform auf europäischer Ebene einrichten. Daher ist geplant, den Expertenrat „Expert Advisory Panel - Health in Health Care Professionals“ im Jahr 2007 zu gründen. Als Vorbereitung hat die Deutsche Suchtstiftung
Matthias Gottschaldt im Herbst 2006 Vertreter aus den unterschiedlichen europäischen Ländern in das Matthias-Gottschaldt-Forum nach Berlin eingeladen, um eine erste Bestandsaufnahme durchzuführen. Hierbei zeigte sich auf europäischer Ebene eine ähnliche Situation wie in Deutschland in den einzelnen Bundesländern. Während in Spanien durch das Engagement einiger weniger Kollegen ein sehr gutes Behandlungsangebot installiert werden konnte (siehe Artikel von Artemann ,Integriertes Versorgungsprogramm für erkrankte Ärzte in Spanien“), gibt es in einigen anderen europäischen Ländern, z.B. Österreich und Schweiz, bis heute noch keine strukturierten Behandlungsangebote, teilweise noch nicht einmal ein Bewusstsein für das Thema Ärztegesundheit.

Neben den Expertenrunden unterstützt die Deutsche Suchtstiftung epidemiologische Untersuchungen zum Thema Ärztegesundheit. Eine erste epidemiologische Totalerhebung wurde in Zusammenarbeit mit der Ärztekammer Hamburg und dem Institut für Allgemeinmedizin in Hamburg durchgeführt. Ein Ergebnis dieser Untersuchung war, dass insbesondere junge Ärztinnen vermehrt Schmerz- und Beruhigungsmittel konsumieren. Dies könnte eine Erklärung dafür sein, dass Mediziner im Vergleich zur Allgemeinbevölkerung ein erhöhtes Risiko für Medikamentenabhängigkeiten haben.

Jüngstes Beispiel für die Unterstützung von Untersuchungen zum Thema Ärztegesundheit ist die Befragung von Psychiatern und Neurologen auf dem Fachkongress der deutschen Psychiatrie, dem DGPPN-Jahreskongress, durch eine Arbeitsgruppe des Universitätsklinikums Ulm unter der Leitung von Frau M. Braun. Erstmals wurden in Deutschland Daten zur Häufigkeit von Burn-Out, Depression und Suchterkrankungen bei deutschen Psychiatern und Nervenärzten erfasst (siehe Artikel von Braun „Burnout, Depression und Substanzgebrauch bei Ärzten - ein Überblick zur derzeitigen Datenlage in Deutschland“).

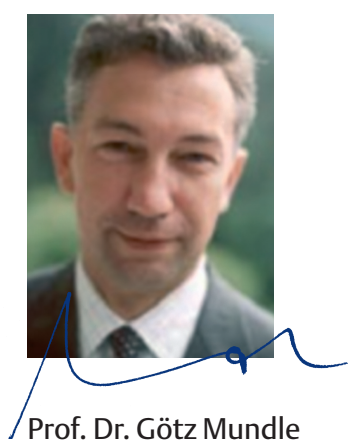

Deutsche Suchtstiftung Matthias Gottschaldt 